\title{
No evidence for MSRV viraemia and glial cell death in acute optic neuritis
}

\author{
A. Petzold* H. Perron ${ }^{\dagger} \quad$ K.T.M. Fernando \\ Gordon T Plant ${ }^{\S}$
}

03.02.2005

*Department of Neuroinflammation, Neuroimmunology Group, Institute of Neurology, Queen Square, London, WC1N 3BG, United Kingdom. Fax: +44 207837 8553, E-mail: a.petzold@ion.ucl.ac.uk

†BioMérieux, R\&D Department, Neurological Programs, Bat.07 Pieèce 112, Chemin de L'Orme, F-69290 Marcy L'Etoile, France

‡Department of Neuroinflammation, MRI Group, Institute of Neurology, Queen Square, London, WC1N 3BG, United Kingdom.

$\S$ The National Hospital of Neurology and Neurosurgery, Department of Neuroophthalmology, Box 93, Queen Square, London, WC1N 3BG, United Kingdom. 
Sir - Viral particles and reverse transcriptase activity have been found in cell culture from patients with multiple sclerosis (MS). ${ }^{1,2}$ Since the first description of MS associated retrovirus (MSRV) in $1989,{ }^{1}$ this has been investigated as a possible cause of $\mathrm{MS}^{3} \mathrm{~A}$ pathogenic link to gliotoxicity has been suggested. ${ }^{4}$ Indeed, a recent study found MSRV in the plasma of a significantly higher proportion (39/39, 100\%) of MS patients compared to patients with other inflammatory neurological diseases $(7 / 10$, $63.6 \%, p=0.004)$ or to healthy blood donors $(5 / 39,12.8 \%)$ from Sardinia, where epidemiological studies revealed an intriguing increase in MS over the past decades. ${ }^{5}$ Moreover, a retrospective study showed that detection of MRSV in CSF had a predictive value for MS patients at disease onset. ${ }^{6}$ It remains unclear whether the presence of MSRV merely represents an epiphenomenon in MS patients or is of aetiological importance.

This study aimed to investigate firstly whether circulating MSRV could be detected in patients with acute optic neuritis as opposed to clinical MS. ${ }^{5}$ Secondly, we hypothesised that those patients with MSRV viraemia may have a higher risk of developing MS compared to those without evidence of infection. Finally we hypothesised that glial cell death associated with actively demyelinating plaques would result in release of astrocytic proteins such as S100B and glial fibrillary acidic protein (GFAP) ${ }^{7}$ and would thus be elevated in those patients who were MSRV positive.

Plasma samples were available from eighteen patients with acute optic neuritis $(\mathrm{ON})$ from a previously reported cohort. ${ }^{8}$ 
Ten patients had baseline MRI scans and 7 had follow-up MRI scans, the remainder had no clinical relapses during the follow up period. The control group consisted of 17 patients with other non-inflammatory neurological diseases. Isoelectric focusing for detection of intrathecal IgG was performed in all of the control patients and none had oligoclonal bands. Blood samples were collected, spun down and stored in $2 \mathrm{~mL}$ aliquots at $-70^{\circ} \mathrm{C}$. Coded samples were analysed for presence of MSRV as described..$^{9}$ The previously defined cut-off for a positive test was an optical density (OD) above 0.8. Plasma S100B and GFAP were measured using standard ELISA systems with a sensitivity of $0.04 \mathrm{ng} / \mathrm{mL}$ and $5 \mathrm{pg} / \mathrm{mL}$, respectively. ${ }^{10,11}$ Statistical analysis was performed using Fisher's exact test for categorical variables and the non-parametric Kruskal-Wallis test for continuous variables.

The patients were younger (median 32 years, range 30-41) compared to the control population $(51,37-61)$, but there was no difference in age between those who developed clinically-definite MS according to the Poser criteria (CDMS) $(27,32-31)$ and those who did not $(33,31-41)$. The median follow-up time was 12 months (range 5-42). As reported previously, ${ }^{8}$ only 2 patients developed CDMS. In another 2 patients the MRI showed new lesions separated in time and space, thus satisfying the MacDonald criteria. ${ }^{12}$ However, none of the patients had evidence of MSRV infection, unlike two of the controls. There was no difference in the median OD, between either the Poser-positive or MacDonald-positive patients. 
The glial proteins S100B and GFAP were not found to be elevated in the plasma of these patients. This may in part be related to the assays' sensitivity.

In contrast to the Sardinian cohort and cell lines from previously reported cohorts, this study investigated for the first time the presence of MSRV in patients with acute ON. ${ }^{1,2,5}$ The working hypothesis that some patients with $\mathrm{ON}$ might have been MSRV carriers with active virion recirculation (viraemia) and have a higher risk of developing MS could not be proven. Interestingly the Sardinian group described infection with MSRV also in a significantly higher proportion of patients with other inflammatory diseases $(63.6 \%)$ when compared to healthy blood donors $(12.8 \%) .{ }^{5} \mathrm{We}$ agree with these authors that infection with MSRV might merely represent an epiphenomenon, ${ }^{5}$ but the very particular type of retroviral agent, belonging to a family of endogenous human retroviruses, HERV-W, raises much more complex questions. ${ }^{13}$

The fact that ON patients did not have negative viraemia on their first symptomatic manifestation, does not preclude them from carrying the MSRV genome in their cells: it may be that it was not expressed prior to and during the onset phase of ON. Further studies should therefore be extended to patients who went on to develop MS, to investigate whether they then had an MSRV viraemia. Such a study has the potential to provide a valuable insight into the possibility that $\mathrm{ON}$ could be caused by another infectious agent or environmental factor, which triggers MSRV reactivation in sus- 
ceptible carriers, as suggested by experimental data on MSRV transactivation. ${ }^{14}$ Whether this could be a condition for the late evolution towards MS in such cases would also require follow-up studies with controls who have not developed MS after acute ON.

This study cannot however contribute to the question of whether MSRV might additionally contribute to gliotoxicity since none of the ON patients had MSRV viraemia.

Acknowledgement The NMR Research Unit is supported by The Multiple Sclerosis Society of Great Britain and Northern Ireland. KF is supported by Biogen.

\section{References}

[1] H Perron, C Geny, A Laurent, et al. Leptomeningeal cell line from multiple sclerosis with reverse transcriptase activity and viral particles. Research in Virology, 140:551-561, 1989.

[2] H Perron, JA Garson, F Bedin, et al. Molecular identification of a novel retrovirus repeatedly isolated from patients with multiple sclerosis. The Collaborative Research Group on Multiple Sclerosis. Proc Natl Acad Sci U S A, 94:7583-7508, 1997.

[3] P Rudge. Does a retrovirally encoded superantigen cause multiple sclerosis? J Neurol Neurosurg Psychiatry, 54:853-805, 1991. 
[4] A Menard, R Amouri, M Michel, et al. Gliotoxicity, reverse transcriptase activity and retroviral RNA in monocyte/macrophage culture supernatants from patients with multiple sclerosis. FEBS Lett, 413:477485, 1997.

[5] A Dolei, C Serra, G Mameli, et al. Multiple sclerosis-associated retrovirus (MSRV) in Sardinian MS patients. Neurology, 58:471-473, 2002.

[6] S Sotgiu, C Serra, G Mameli, et al. Multiple Sclerosis-associated retrovirus and MS prognosis: an observational study. Neurology, 59:1071-1073, 2002.

[7] A Petzold, MJ Eikelenboom, D Gveric, G Keir, M Chapman, RH Lazeron, et al. Markers for different glial cell responses in multiple sclerosis: Clinical and pathological correlations. Brain, 125:1462-1473, 2002.

[8] A Petzold, K Rejdak, and GT Plant. Axonal degeneration and inflammation in acute optic neuritis. J Neurol Neurosurg Psychiatry, 75:1178-1180, 2004.

[9] JA Garson, PW Tuke, P Giraud, G Paranhos-Baccala, and H Perron. Detection of virion-associated MSRV-RNA in serum of patients with multiple sclerosis. The Lancet, 351:33, 1998. 
[10] AJE Green, G Keir, and EJ Thompson. A specific and sensitive ELISA for measuring S-100b in cerebrospinal fluid. J Immunol Meth, 205:3541, 1997.

[11] A Petzold, G Keir, Green AJE, G Giovannoni, and EJ Thompson. An ELISA for glial fibrillary acidic protein. J Immunol Meth, 287:169-177, 2004.

[12] WI McDonald, A Compston, G Edan, et al. Recommended diagnostic criteria for multiple sclerosis: guidelines from the International Panel on the diagnosis of multiple sclerosis. Ann Neurol, 50:121-127, 2001.

[13] H Perron, JP Perin, F Rieger, and PM Alliel. Particle-associated retroviral RNA and tandem RGH/HERV-W copies on human chromosome 7q: possible components of a 'chain-reaction' triggered by infectious agents in multiple sclerosis? J Neurovirol, 6 Suppl 2:S67-75, 2000.

[14] H Perron, M Suh, B Lalande, B Gratacap, A Laurent, P Stoebner, and JM Seigneurin. Herpes simplex virus ICPO and ICP4 immediate early proteins strongly enhance expression of a retrovirus harboured by a leptomeningeal cell line from a patient with multiple sclerosis. $J$ Gen Virol, 74:65-72, 1993. 


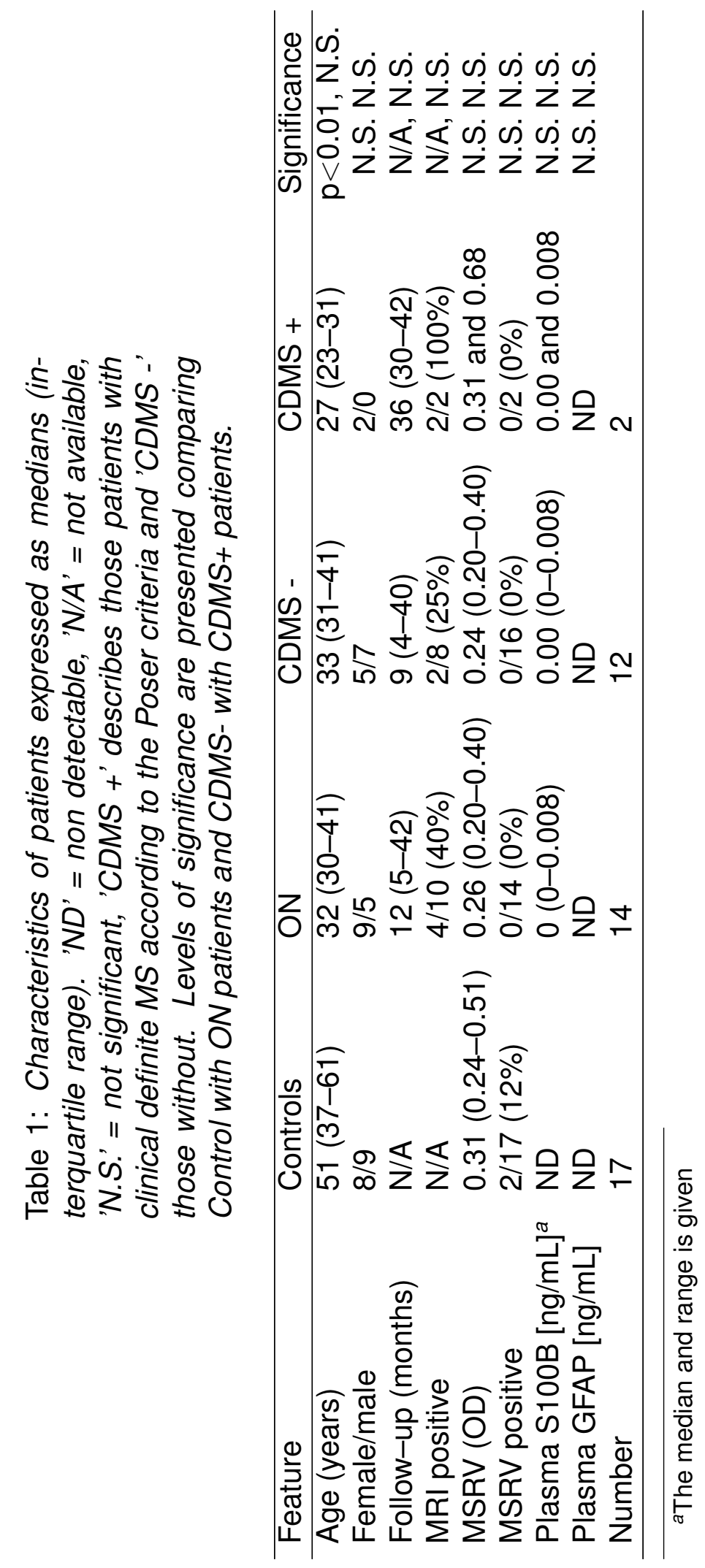

